\title{
Activating FGFR1 Mutations in Sporadic Pheochromocytomas
}

\author{
Jenny Welander ${ }^{1} \cdot$ Małgorzata Lysiak $^{1} \cdot$ Michael Brauckhoff $^{2,3} \cdot$ Laurent Brunaud $^{4}$. \\ Peter Söderkvist $^{1} \cdot$ Oliver Gimm ${ }^{1,5}$
}

Published online: 20 November 2017

(C) The Author(s) 2017. This article is an open access publication

\begin{abstract}
Introduction Pheochromocytomas are neuroendocrine tumors of the adrenal glands. Up to $40 \%$ of the cases are caused by germline mutations in one of at least 15 susceptibility genes, making them the human neoplasms with the highest degree of heritability. Recurrent somatic alterations are found in about $50 \%$ of the more common sporadic tumors with $N F 1$ being the most common mutated gene (20-25\%). In many sporadic tumors, however, a genetic explanation is still lacking.

Materials and methods We investigated the genomic landscape of sporadic pheochromocytomas with whole-exome sequencing of 16 paired tumor and normal DNA samples and extended confirmation analysis in 2 additional cohorts comprising a total of 80 sporadic pheochromocytomas.

Results We discovered on average 33 non-silent somatic variants per tumor. One of the recurrently mutated genes was FGFR1, encoding the fibroblast growth factor receptor 1, which was recently revealed as an oncogene in pediatric brain tumors. Including a subsequent analysis of a larger cohort, activating FGFRl mutations were detected in three of 80 sporadic pheochromocytomas (3.8\%). Gene expression microarray profiling showed that these tumors clustered with NF1-, RET,- and HRAS-mutated pheochromocytomas, indicating activation of the MAPK and PI3KAKT signal transduction pathways.

Conclusion Besides RET and HRAS, FGFRl is only the third protooncogene found to be recurrently mutated in pheochromocytomas. The results advance our biological understanding of pheochromocytoma and suggest that somatic FGFRl activation is an important event in a subset of sporadic pheochromocytomas.
\end{abstract}

Electronic supplementary material The online version of this article (https://doi.org/10.1007/s00268-017-4320-0) contains supplementary material, which is available to authorized users.

Peter Söderkvist and Oliver Gimm have contributed equally to this manuscript.

Peter Söderkvist

peter.soderkvist@liu.se

1 Department of Clinical and Experimental Medicine, Faculty of Medicine and Health Sciences, Linköping University, 58185 Linköping, Sweden

2 Department of Surgery, Haukeland University Hospital, 5021 Bergen, Norway

\section{Introduction}

Pheochromocytomas (PCCs) are tumors arising from the neural crest-derived cells of the adrenal medulla, and paragangliomas (PGLs) are their extra-adrenal

Department of Clinical Science, University of Bergen, 5020 Bergen, Norway

4 Department of Digestive, Hepato-Biliary and Endocrine Surgery, CHU Nancy - Hospital Brabois Adultes, University de Lorraine, 54511 Vandoeuvre-les-Nancy, France

5 Department of Surgery, County Council of Östergötland, 58185 Linköping, Sweden 
counterparts. They may cause hypertension due to overproduction of catecholamines, with symptoms including frequent episodes of headache, palpitations and sweating, and an increased risk of cardiovascular disease [1]. PCCs and PGLs have a highly diverse genetic background; up to $40 \%$ of the cases are caused by germline mutations in one of at least 15 so far identified susceptibility genes, making them the human neoplasms with the highest degree of heritability $[1,2]$. More and more is also known regarding alterations in the sporadic tumors. During the past few years, somatic mutations have been revealed in several of the genes known from the hereditary cases, most frequently in NF1 [3, 4]. HRAS was found as the first gene with recurrent somatic mutations that is not associated with hereditary PCCs (6), and more recently, frequent somatic mutations were also discovered in ATRX [5, 6]. Initially, gene expression profiling showed that PCCs could be divided into at least two different clusters based on their expression signature [7]. The first cluster contains tumors with $V H L, S D H x$, and EPASI mutations and displays mRNA expression associated with the hypoxic response. The second cluster contains tumors with RET, NFl, TMEM127, and MAX mutations and is enriched for mRNA expression related to activation of kinase signaling cascades. Recently, third and fourth clusters have been described [8]. The third cluster contains tumors with somatic MAML3 fusion genes, CSDE1 and ATRX mutations leading to activation of the Wnt signaling. The fourth cluster contains genes known to be adrenal cortex markers $(C Y P 11 B 2, C Y P 21 A 2$, and STAR) and is associated with the presence of cortical cells and termed cortical admixture. Despite this remarkable progress in the field, a large portion of the sporadic tumors still remains without any known genetic driver event $[9,10]$. We therefore hypothesized that there are additional targets of recurrent somatic mutations in PCCs. To examine this, we performed wholeexome sequencing of paired tumor and normal DNA from patients with sporadic PCC and followed up the results by studying whole-transcriptome gene expression.

\section{Materials and methods}

\section{Patients and samples}

The discovery cohort of this study consisted of 16 PCCs with corresponding peripheral blood samples from 16 patients operated at Linköping University Hospital, Sweden (Table S1). Findings were further investigated in 15 remaining PCCs from Sweden and Norway with known mutational status in PCC-associated genes (Table S1), followed by 49 additional sporadic PCCs from Hôpital de Brabois, Nancy, France (Table S2). All patients had been diagnosed with sporadic PCC due to absence of family history and syndromic presentation. Two sporadic PCCs and ten tumors from patients with hereditary syndromes were also available and tested (Table S2). Informed consent was obtained from all participants, and the local ethic committees approved the study.

\section{Methods}

All methods are described in the supplementary material.

\section{Results}

\section{The mutation landscape of pheochromocytomas}

We performed whole-exome sequencing of 16 tumors with paired constitutional DNA from patients with apparently sporadic disease (Table S1). Six of the cases had previously tested negative for mutations in known PCC-related genes $[4,9]$. Sequencing generated an average depth of $119 \times$ for tumor samples and $97 \times$ for blood samples, with a depth of at least $10 \times$ in 97.9 and $97.5 \%$ of the exome, respectively. When investigating somatic mutations in the previously known genes, we detected truncating NF1 mutations in three of the tumors with previously unknown mutation status, as could be expected in the light of earlier studies $[3,4]$. In addition, somatic mutations in the previously known genes VHL, RET, MAX, and HRAS were found in one tumor each (Table S3), in agreement with frequencies in previous reports (Table 1) [7, 10-13]. Despite the premises, we did not find any ATRX mutations [5, 6]. Moreover, we investigated germline alterations and detected a germline frameshift mutation in $S D H B$ in one case for which the mutation status was previously unknown (Table S3). Germline missense variants were also found in $E G L N 2, K I F 1 B \beta, N F 1$, and $S D H D$, but bioinformatic analysis suggests that these are either benign or of unknown significance for the disease (Table S3).

We detected totally 542 non-silent somatic variants in the cohort of 16 tumors. The median number per tumor was 33 non-silent somatic variants, with a range from 16 to 60 per tumor. Most of the detected alterations were unique to one tumor, but some genes harbored non-silent somatic mutations in two or more samples, including the NF1 gene as could be expected. The recurrently altered genes also included FGFRl, in which activating point mutations were recently discovered in pediatric pilocytic astrocytoma [14]. 


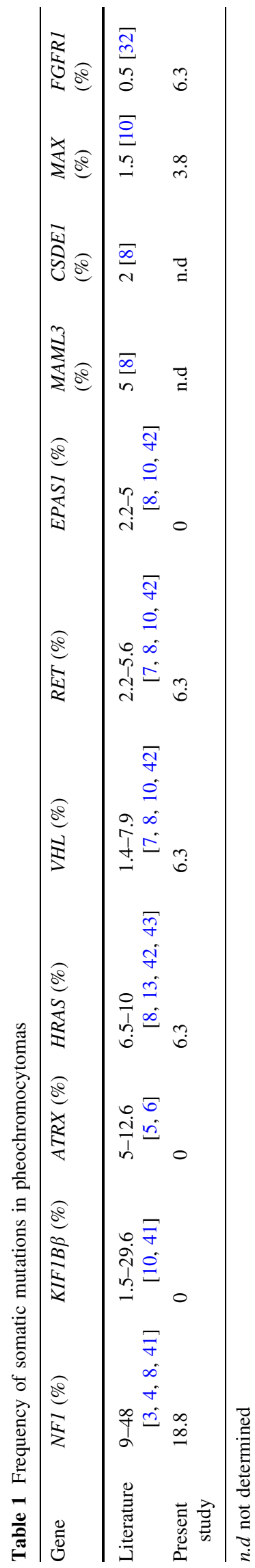

\section{Recurrent hotspot FGFR1 mutations}

We detected two somatic FGFR1 mutations, Asn546Lys (c.1638C $>$ A) and Lys656Glu (c.1966A $>$ G) (Figs. 1 and 2). They exactly correspond to the two hotspot sites for activating mutations recently discovered in pediatric brain tumors [14]. To investigate the prevalence of FGFRI mutations in sporadic PCCs, we performed Sanger sequencing of the three most common hotspots in FGFRI (Fig. S1) in 15 remaining tumors from the Scandinavian cohort (Table S1) and detected one additional Asn546Lys mutation (Fig. 2). Subsequent analysis of 49 sporadic PCCs from France (Table S2) did not reveal any additional mutations (Fig. 2). In total, the combined cohort consisted of 80 apparently sporadic PCCs, of which three tumors (samples \#40, \#50, \#64; 3.8\%) carried FGFR1 mutations
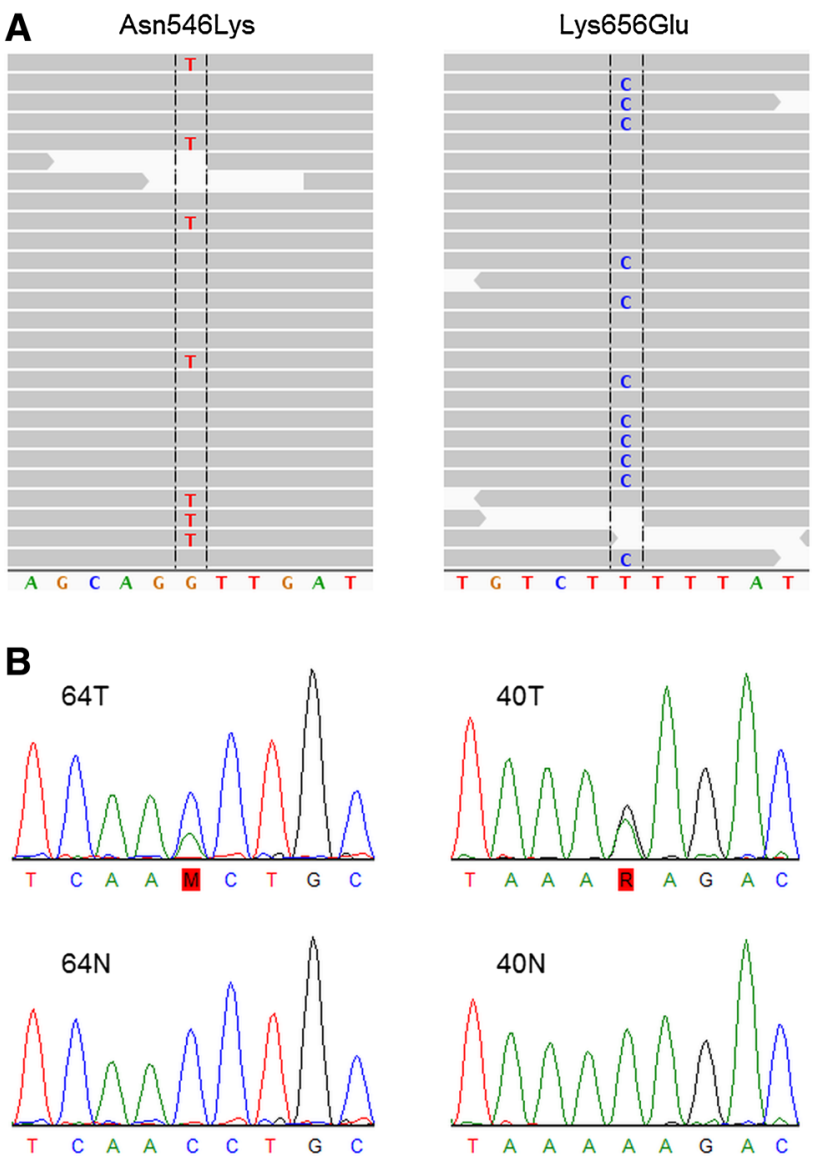

Fig. 1 Somatic hotspot mutations in FGFR1. a Overview of nextgeneration sequencing reads from the mutated sites in Integrative Genomics Viewer. Read bases that match the ho19 reference are displayed in gray, and mismatches are indicated with color coded alternate alleles (FGFRl is oriented on the reverse strand; hence, the sequence is here the reverse complement to the transcribed sequence). b Validation of the mutations in the two tumor samples (64T and 40T) with Sanger sequencing (in the direction of transcription) and the corresponding sequences from blood samples 
Fig. 2 Frequency of FGFR1 mutations indifferent cohorts. Flow diagram simplifying the process of initial discovery and validation of somatic FGFR1 mutations in sporadic pheochromocytomas (PCCs). * With regard to somatic FGFRI mutations; \# of somatic FGFR1 mutations

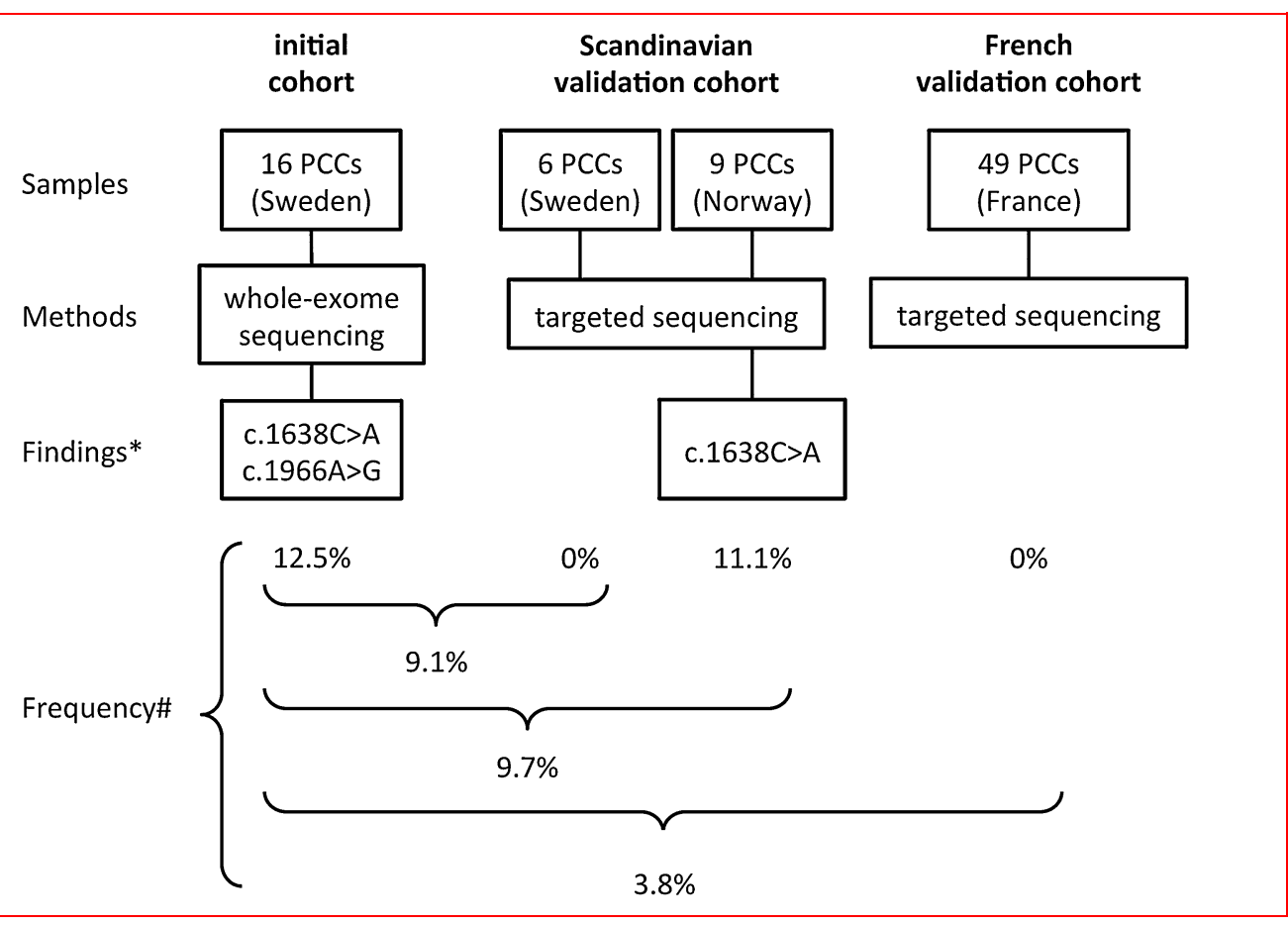

Table 2 Clinical and genetic data for cases with somatic FGFR1 mutations

\begin{tabular}{llllllll}
\hline Case ID & Gender & Age (years) & Tumor size $(\mathrm{mm})$ & Malignancy & Mutation $^{\text {a }}$ & Protein alteration & Copy number \\
\hline 40 & Female & 63 & 32 & Benign & c.1638C $>$ A & Asn546Lys & Gain \\
50 & Female & 80 & 10 & Benign & c.1966A $>$ G & Lys656Glu & Normal \\
64 & Male & 56 & 32 & Benign & c.1638C $>$ A & Asn546Lys & Normal \\
\hline
\end{tabular}

${ }^{\mathrm{a}}$ Mutations were annotated according to the Ensembl transcript ENST00000447712

(Fig. 2). The cases with somatic FGFRl mutations were two females and one male with a mean age of 66 years (Table 2). One of the FGFR1-mutated tumors also had a somatic MAX mutation, whereas the other two did not have any mutations in known PCC-associated genes (Fig. S2). Apart from the main cohort of sporadic PCCs, we tested also two sporadic PGLs and ten hereditary PCCs (four MEN2B, two VHL, three PGL1, and one PGL4), yet no FGFR I mutations were detected. In addition, we searched samples with available RNA (Table $\mathrm{S} 4$ ) for a known fusion gene involving FGFRl, i.e., FGFRl-TACC1 [15, 16], which is known to play an oncogenic role in glioblastomas, but no gene fusions were found in PCCs.

\section{Copy number gains at the FGFR1 locus}

Copy number analysis showed a gain of FGFRl in four of 80 sporadic PCCs, one of which also had a FGFRl mutation (Fig. S3). SNP microarray data from a previous study [4] were used to verify these findings where possible. For the sample with both FGFRI copy gain and FGFRI mutation, SNP microarray data were available and amplification of the FGFR1 locus was confirmed and microarray data showed that it was part of a $\sim 2.5 \mathrm{Mb}$ amplification unit on chromosome 8p (Fig. S4). Copy number loss in the FGFR1 region was seen in 11/91 sporadic PCCs (Table S4), none of which carried any FGFR1 mutations. For three of four cases with loss and where microarray data were also available, the loss could be seen to cover large parts of chromosome 8, suggesting that loss of FGFR1 in these cases is a passenger event (for the fourth sample, a loss was not detectable using the array technique, possibly because of a too low marker resolution).

\section{Gene expression profiling of FGFR1-mutated tumors}

Global gene expression analysis showed that the three PCCs with FGFR1 mutations clustered in "cluster 2" together with tumors that contained mutations in RET, $N F 1$, and HRAS. This indicates that FGFRl mutations are associated with activation of kinase signal transduction 
pathways, including the MAPK and PI3K-AKT pathways [9]. Among all the genes that passed quality control, there were no genes that differed significantly in expression between tumors with and without FGFRI mutations after correction for multiple testing. The reasons for this may be that the FGFRl-mutated tumors have a gene expression profile very similar to the other tumors in "cluster 2 " and that the number of tested FGFRl-mutated tumors was too small to detect minor differences in expression patterns. There was no association between FGFRl mutations and FGFRl gene expression, as would be expected since the mutations are thought to have their effect on the protein level. However, copy gain of FGFR1 was associated with an enhanced FGFRl gene expression (Fig. S5), suggesting a gene-dose effect.

\section{Analysis of other fibroblast growth factor receptors}

To further extend the knowledge of fibroblast growth factor receptors in PCCs, exons containing hotspot regions in FGFR2 and FGFR3 (Fig. S1) were investigated for mutations in all samples (sporadic and hereditary), but no mutations were detected. A very rare polymorphism in FGFR3 (rs17881656, Phe384Leu) was detected in three cases (two of which were hereditary) and was also present in normal DNA of the two patients where blood was available (Table S4). The variant was predicted as benign using the Polyphen-2, SIFT, and PROVEAN algorithms [17-19]. Allele frequency was then analyzed in a regional healthy control population $(n=739)$, as well as checked in the Swedish 1000 Genomes population ( $n=1000$; https:// swegen-exac.nbis.se/) and the ExAC database (http://exac. broadinstitute.org/). The C-allele frequency was $1.6 \%$ in the PCC-cohort and $0.34,0.4$ and $0.31 \%$ in the regional control cohort, the Swedish 1000 Genome database and the ExAC database, respectively. Two-tailed Fisher's exact test showed a borderline significant difference when compared to the Swedish control population $(p=0.049)$, the SweGen database $(p=0.07)$, and the ExAC database $(p=0.02)$. A rare germline variant in FGFRl was detected in one case (c. 381T $>$ G, Asp127Glu, rs750795714) and reported in only 2 out of 120,880 alleles in the ExAC database. It was outside of the mutational hotspots and was predicted as benign by PolyPhen. The known gene fusion involving FGFR3 (FGFR3-TACC3 [15, 16]) was also investigated, but no gene fusions were discovered. Available DNA microarray data from 21 tumors did not reveal any copy number alterations in any of the FGFRI-3 genes other than FGFRl. A summary of FGFRI-3 sequence variations, copy number alterations, and gene expression levels for all samples is given in Table S4.

\section{Discussion}

In this report, we present evidence that activating mutations in a gene encoding for fibroblast growth factor receptor 1, FGFR1, are important somatic events in some sporadic PCCs. FGFRs, which in humans include FGFR1 to FGFR4, are receptor tyrosine kinases that are involved in multiple processes during embryonic development, including mesenchymal-epithelial communication and formation of several organ systems [20, 21]. In the adult, fibroblast growth factor receptor signaling is involved in tissue homeostasis and regulates processes such as tissue repair, angiogenesis, and inflammation. Binding of FGFs to FGFRs induces receptor dimerization that activates the intracellular kinase domain and leads to transphosphorylation [21]. The phosphorylated tyrosine residues function as docking sites for adaptor proteins, for example FRS2, which can be phosphorylated by FGFRs. This leads to an activation of several signal transduction pathways, including the RAS-RAF-MAPK and PI3K-AKT pathways. The most established link between FGFRs and cancer is probably with bladder cancer, in which FGFR3 is one of the most commonly mutated genes [22] and the mutations are associated with a non-invasive behavior [23]. Mutations in FGFR2 and FGFR3 have also been reported in other tumor forms, whereas activation of FGFR1 has mainly been observed in the form of FGFR1 amplification in breast and lung cancer [20]. However, occasional point mutations and fusion genes involving FGFRl have been observed in glioblastomas [24], which is in agreement with FGFR1 being the most abundant fibroblast growth factor receptor in the nervous system [25]. Most recently, frequent $F G F R 1$ mutations in two hotspot sites were revealed in another brain tumor: pilocytic astrocytoma [14]. The two variants detected in this study, Asn546Lys and Lys656Glu, affect the kinase domain and have been shown to promote cell proliferation [26], and Asn546Lys also alter FGFR1 autophosphorylation [27]. Furthermore, overexpression of FGFR1 results in MAPK and AKT activation and neurite outgrowth in the rat pheochromocytoma cell line PC12 [25].

In this study, we discovered that the same FGFR1 hotspot residues as in certain brain tumors, Asn546 and Lys656 (also reported in 1 of 394 PCCs in the COSMIC database [28]), are recurrently mutated in PCCs. Interestingly, the first cohort investigated in this study, which consisted of Scandinavian cases, had a prevalence of FGFRl mutations of almost $10 \%$. However, further investigation in a French cohort did not reveal any additional mutations, resulting in a total frequency of 3.8\% (Fig. 2) in the combined cohorts of 80 sporadic PCCs. This means that somatic FGFRI mutations are a rare but 
Fig. 3 Hierarchical clustering

of tumors based on gene

expression levels. Mutation

status is indicated below the

dendrogram, showing that

tumors with FGFR1 mutations

cluster together with those that

have RET, NF1, or HRAS

mutations. Whereas mutations

in the known susceptibility

genes were mutually exclusive,

one mutation in the novel

susceptibility gene, FGFRl,

occurred in combination with a

somatic MAX mutation

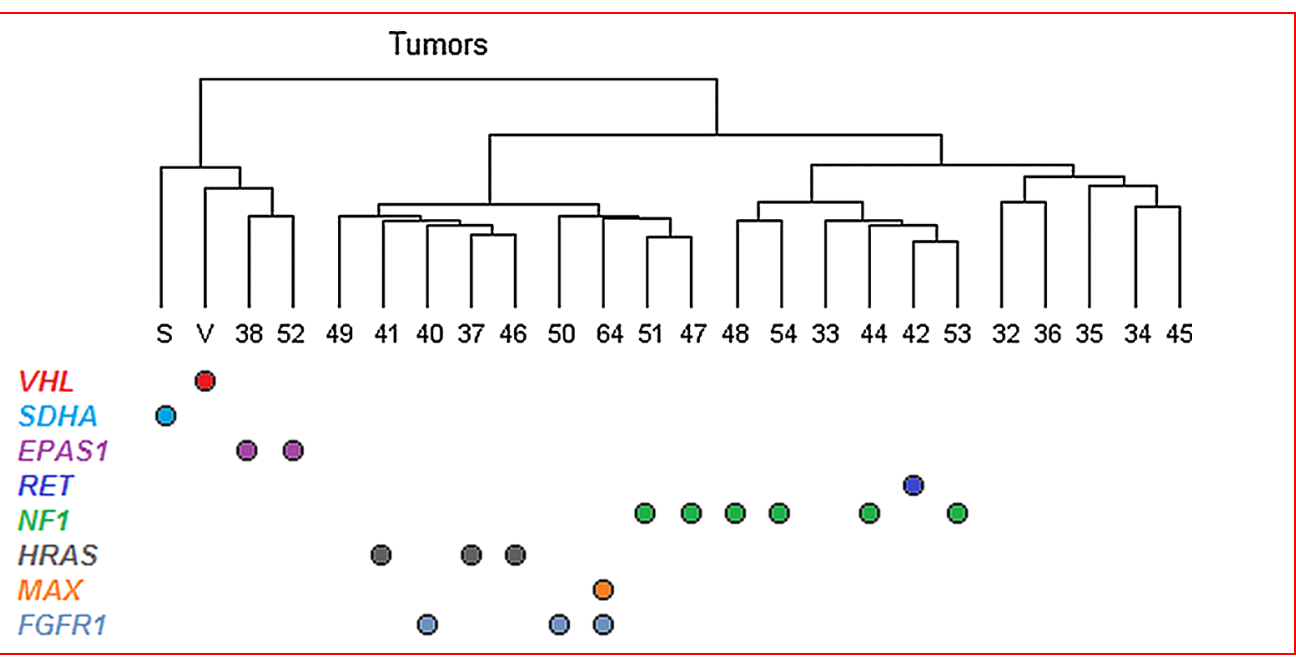

recurrent event in PCCs (Table 1). The difference in the frequency of somatic FGFRl mutations between PCCs from different countries may be due to random variation, or, alternatively, there could be regional differences in the frequency of mutations. Data on the ethnic background are not available in our cohort. Possibly, regional differences in the occurrence of certain germline variants could alter the predisposition to obtain somatic FGFRl mutations. Such a phenomenon has previously been observed for a polymorphism that is associated with a hypermutability of the $A P C$ gene [29] involved in colorectal cancer. The significance of FGFR1 mutations in PCCs is also supported by data from the TCGA cohort of PCCs and PGLs (http:// cancergenome.nih.gov/). Analyzed sequencing data accessed through cBioPortal $[30,31])$ displayed a prevalence of Asn546Lys mutation (corresponding to the Asn577Lys in the cBioPortal) in $2 / 173(1.2 \%)$ of the samples. Interestingly, Toledo et al. very recently found the Asn546Lys mutation in 1/43 PCCs/PGLs, but none in a 136 patient validation cohort supporting the role of FGFR1 mutation in PCCs/PGLs as a rare but recurrent event in three different cohorts at a frequency similar to several other PCC/PGL susceptibility genes [32]. In addition, we observed copy number gains in the $F G F R I$ region, which were associated with an increased FGFRl gene expression, which is in agreement with an oncogenic role of FGFRl in PCCs. Our findings constitute an additional PCC susceptibility gene associated with MAPK and AKT activation, and indeed, gene expression profiling showed that the FGFRI-mutated tumors clustered together with tumors having mutations in the NF1, RET, and HRAS genes (Fig. 3). Future studies in larger cohorts will be valuable to more accurately estimate the frequency and the potential clinical consequences of FGFRl alterations in PCCs/PGLs. The potential association of the FGFR3 variant Phe384Leu (rs17881656) with PCCs warrants further examination.
The whole-exome sequencing approach used in this study also detected a median of 33 non-silent somatic mutations in each tumor. Most of the altered genes detected here were only altered in a single sample, suggesting that there is a large diversity of somatic events occurring in PCCs. Many of these may be passenger events, and further studies with larger sample sizes will probably be required in order to identify additional true but infrequent drivers in the tumorigenesis [33]. In agreement with previous reports $[34,35]$, the exome sequencing technique was also useful in order to find a germline $S D H B$ mutation in a patient with apparently sporadic PCCs, but we also detected numerous variants of unknown significance that may be difficult to interpret in a clinical setting. For gene and pathway discovery, however, next-generation sequencing and omics approaches have been incredibly successful [34, 36-38] and will likely result in the detection of additional PCC/ PGL-associated genes in the near future. Indeed, recent exome sequencing initiatives published during the finishing of this work have revealed additional genes, including $A T R X, M D H 2$, and $K M T 2 D$, to be potentially involved in PCC/PGL pathogenesis [5, 6, 39, 40], and FGFRl can now be added to the list of PCC susceptibility genes. This finding advances our biological understanding of PCC development and possibly opens for novel therapeutic options involving FGFR inhibitors, which are currently being evaluated in other tumor forms [21].

Acknowledgements The authors would like to acknowledge the support from FORSS (Medical Research Council of Southeast Sweden), Science for Life Laboratory, the National Genomics Infrastructure (NGI), Sweden, the Knut and Alice Wallenberg Foundation and UPPMAX for providing assistance in massive parallel sequencing and computational infrastructure. We also thank Miłosz Roliński, Ida Gustavsson, Cassandra Ekman, Annette Molbaek, and Åsa Schippert for valuable technical support. This work was supported by Grants from Linköping University, the Swedish Cancer Society, FORSS and LiU Cancer. 


\section{Compliance with ethical standards}

Conflict of interest The authors have no conflict of interest.

Open Access This article is distributed under the terms of the Creative Commons Attribution 4.0 International License (http://crea tivecommons.org/licenses/by/4.0/), which permits unrestricted use, distribution, and reproduction in any medium, provided you give appropriate credit to the original author(s) and the source, provide a link to the Creative Commons license, and indicate if changes were made.

\section{References}

1. Dahia PL (2014) Pheochromocytoma and paraganglioma pathogenesis: learning from genetic heterogeneity. Nat Rev Cancer 14:108-119

2. Favier J, Amar L, Gimenez-Roqueplo AP (2015) Paraganglioma and phaeochromocytoma: from genetics to personalized medicine. Nat Rev Endocrinol 11:101-111

3. Burnichon N, Buffet A, Parfait B et al (2012) Somatic NF1 inactivation is a frequent event in sporadic pheochromocytoma. Hum Mol Genet 21:5397-5405

4. Welander J, Larsson C, Backdahl M et al (2012) Integrative genomics reveals frequent somatic NF1 mutations in sporadic pheochromocytomas. Hum Mol Genet 21:5406-5416

5. Fishbein L, Khare S, Wubbenhorst B et al (2015) Whole-exome sequencing identifies somatic ATRX mutations in pheochromocytomas and paragangliomas. Nat Commun 6:6140

6. Flynn A, Benn D, Clifton-Bligh R et al (2015) The genomic landscape of phaeochromocytoma. J Pathol 236:78-89

7. Burnichon N, Vescovo L, Amar L et al (2011) Integrative genomic analysis reveals somatic mutations in pheochromocytoma and paraganglioma. Hum Mol Genet 20:3974-3985

8. Fishbein L, Leshchiner I, Walter V et al (2017) Comprehensive Molecular Characterization of Pheochromocytoma and Paraganglioma. Cancer Cell 31:181-193

9. Welander J, Andreasson A, Brauckhoff M et al (2014) Frequent EPAS1/HIF2alpha exons 9 and 12 mutations in non-familial pheochromocytoma. Endocr Relat Cancer 21:495-504

10. Welander J, Andreasson A, Juhlin CC et al (2014) Rare germline mutations identified by targeted next-generation sequencing of susceptibility genes in pheochromocytoma and paraganglioma. J Clin Endocrinol Metab 99:E1352-1360

11. Crona J, Delgado Verdugo A, Maharjan R et al (2013) Somatic mutations in H-RAS in sporadic pheochromocytoma and paraganglioma identified by exome sequencing. J Clin Endocrinol Metab 98:E1266-1271

12. Burnichon N, Cascon A, Schiavi F et al (2012) MAX mutations cause hereditary and sporadic pheochromocytoma and paraganglioma. Clin Cancer Res 18:2828-2837

13. Oudijk L, de Krijger RR, Rapa I et al (2014) H-RAS mutations are restricted to sporadic pheochromocytomas lacking specific clinical or pathological features: data from a multi-institutional series. J Clin Endocrinol Metab 99:E1376-1380

14. Jones DT, Hutter B, Jager N et al (2013) Recurrent somatic alterations of FGFR1 and NTRK2 in pilocytic astrocytoma. Nat Genet 45:927-932

15. Singh D, Chan JM, Zoppoli P et al (2012) Transforming fusions of FGFR and TACC genes in human glioblastoma. Science $337: 1231-1235$
16. Di Stefano AL, Fucci A, Frattini V et al (2015) Detection, Characterization, and Inhibition of FGFR-TACC Fusions in IDH Wild-type Glioma. Clin Cancer Res 21:3307-3317

17. Adzhubei IA, Schmidt S, Peshkin L et al (2010) A method and server for predicting damaging missense mutations. Nat Methods 7:248-249

18. Kumar P, Henikoff S, Ng PC (2009) Predicting the effects of coding non-synonymous variants on protein function using the SIFT algorithm. Nat Protoc 4:1073-1081

19. Choi Y, Sims GE, Murphy S et al (2012) Predicting the functional effect of amino acid substitutions and indels. PLoS One 7:e46688

20. Haugsten EM, Wiedlocha A, Olsnes S et al (2010) Roles of fibroblast growth factor receptors in carcinogenesis. Mol Cancer Res 8:1439-1452

21. Turner N, Grose R (2010) Fibroblast growth factor signalling: from development to cancer. Nat Rev Cancer 10:116-129

22. Cheng L, Zhang S, Davidson DD et al (2009) Molecular determinants of tumor recurrence in the urinary bladder. Future Oncol $5: 843-857$

23. Hernandez S, Lopez-Knowles E, Lloreta J et al (2006) Prospective study of FGFR3 mutations as a prognostic factor in nonmuscle invasive urothelial bladder carcinomas. J Clin Oncol 24:3664-3671

24. The_Cancer_Genome_Atlas_Research_Network (2008) Comprehensive genomic characterization defines human glioblastoma genes and core pathways. Nature 455:1061-1068

25. Hausott B, Schlick B, Vallant N et al (2008) Promotion of neurite outgrowth by fibroblast growth factor receptor 1 overexpression and lysosomal inhibition of receptor degradation in pheochromocytoma cells and adult sensory neurons. Neuroscience 153:461-473

26. Yoon K, Nery S, Rutlin ML et al (2004) Fibroblast growth factor receptor signaling promotes radial glial identity and interacts with Notch1 signaling in telencephalic progenitors. J Neurosci 24:9497-9506

27. Lew ED, Furdui CM, Anderson KS et al (2009) The precise sequence of FGF receptor autophosphorylation is kinetically driven and is disrupted by oncogenic mutations. Sci Signal 2:6

28. Forbes SA, Bindal N, Bamford S et al (2011) COSMIC: mining complete cancer genomes in the Catalogue of Somatic Mutations in Cancer. Nucleic Acids Res 39:D945-950

29. Laken SJ, Petersen GM, Gruber SB et al (1997) Familial colorectal cancer in Ashkenazim due to a hypermutable tract in APC. Nat Genet 17:79-83

30. Gao J, Aksoy BA, Dogrusoz U et al (2013) Integrative analysis of complex cancer genomics and clinical profiles using the cBioPortal. Sci Signal 6:11

31. Cerami E, Gao J, Dogrusoz U et al (2012) The cBio cancer genomics portal: an open platform for exploring multidimensional cancer genomics data. Cancer Discov 2:401-404

32. Toledo RA, Qin Y, Cheng ZM et al (2016) Recurrent mutations of chromatin-remodeling genes and kinase receptors in pheochromocytomas and paragangliomas. Clin Cancer Res 22:2301-2310

33. Lawrence MS, Stojanov P, Mermel CH et al (2014) Discovery and saturation analysis of cancer genes across 21 tumour types. Nature 505:495-501

34. Crona J, Verdugo AD, Granberg D et al (2013) Next-generation sequencing in the clinical genetic screening of patients with pheochromocytoma and paraganglioma. Endocr Connect 2:104-111

35. McInerney-Leo AM, Marshall MS, Gardiner B et al (2014) Whole exome sequencing is an efficient and sensitive method for detection of germline mutations in patients with phaeochromcytomas and paragangliomas. Clin Endocrinol (Oxf) 80:25-33 
36. Comino-Mendez I, Gracia-Aznarez FJ, Schiavi F et al (2011) Exome sequencing identifies MAX mutations as a cause of hereditary pheochromocytoma. Nat Genet 43:663-667

37. Letouze E, Martinelli C, Loriot C et al (2013) SDH mutations establish a hypermethylator phenotype in paraganglioma. Cancer Cell 23:739-752

38. Toledo RA, Qin Y, Srikantan S et al (2013) In vivo and in vitro oncogenic effects of HIF2A mutations in pheochromocytomas and paragangliomas. Endocr Relat Cancer 20:349-359

39. Cascon A, Comino-Mendez I, Curras-Freixes M, et al. (2015) Whole-exome sequencing identifies $\mathrm{MDH} 2$ as a new familial paraganglioma gene. J Natl Cancer Inst. https://doi.org/10.1093/ jnci/djv053
40. Juhlin CC, Goh G, Healy JM et al (2015) Whole-exome sequencing characterizes the landscape of somatic mutations and copy number alterations in adrenocortical carcinoma. J Clin Endocrinol Metab 100:E493-502

41. Evenepoel L, Helaers R, Vroonen L et al (2017) KIF1B and NF1 are the most frequently mutated genes in paraganglioma and pheochromocytoma tumors. Endocr Relat Cancer 24:L57-L61

42. Crona J, Nordling M, Maharjan R et al (2014) Integrative genetic characterization and phenotype correlations in pheochromocytoma and paraganglioma tumours. PLoS One 9:e86756

43. Stenman A, Welander J, Gustavsson I et al (2016) HRAS mutation prevalence and associated expression patterns in pheochromocytoma. Genes Chromosomes Cancer 55:452-459 